\title{
Un vistazo a la arquitectura de un sistema inteligente de administración de inventarios basado en tecnologías de la Industria 4.0
}

\author{
The architecture of an intelligent inventory management system based on Industry 4.0 \\ technologies: a glance
}

\author{
Briggit Ortega-Palma ${ }^{a}$, Aline Vera-Márquez ${ }^{b}$, Ruth Hernández-García ${ }^{c}$, Isidro J. González- \\ Hernández ${ }^{d}$, Jorge Zuno-Silva ${ }^{e}$
}

\begin{abstract}
:
Today, companies are facing a global, technological, and competitive market (commerce through e-commerce and Industry 4.0). Due to this competition, supply chain management represents one of the most important factors in the profitability of a business. Among the different links in the supply network, warehouses are an essential component in the chain and are increasingly seen as strategic facilities to provide competitive advantages. The operational challenges in inventory control and management in complex operating environments are due to the uncertainty or randomness of the various interlocking business processes. To face these challenges, incorporating emerging Industry 4.0 technologies into logistics and supply chain processes can actively contribute to accelerating solutions related to warehouse operations and management. In this manuscript, is presented qualitative research, of a bibliographic and documentary nature, to identify the main components of an architecture for an intelligent inventory management system based on Industry 4.0 technologies. Research shows that architecture is based on two major technologies, Internet of Things and Rad io Frequency Identification systems. It was also identified that the majority of intelligent systems based on Internet of Things, must consider 5 layers to manage and control inventory intelligently.
\end{abstract}

Keywords:

Inventory management, Internet of Things, RFID

\section{Resumen:}

Hoy en día, las empresas se enfrentan a un mercado más global, tecnológico y competitivo (el comercio a través del e-comercio y la Ind ustria 4.0). Debido a esta competencia, la administración de la cadena de suministro representa uno de los factores más importantes en la rentabilidad de un negocio. Entre los diferentes eslabones de la cadena de abastecimiento, los almacenes son un componente esencial en la red de suministro y se consideran cada vez más como instalaciones estratégicas para proporcionar ventajas competitivas. Las dificultades para administrar y controlar el inventario en entornos operativos complejos se debe a la incertidumbre o ale atoriedad de los diversos procesos comerciales entrelazados. Para enfrentar estos desafíos, la incorporación de tecnologías emergentes de la Industria 4.0 en los procesos de logística y cadena de suministro pueden contribuir activamente a acelerar las soluciones relacionadas con las operaciones y la administración de los almacenes. En este trabajo, se presenta una investigación cualitativa, de carácter bibliográfico y documental para identificar los principales componentes de una arquitectura para un sistema inteligente de administración de inventarios basado en tecnologías de la Industria 4.0. La investigación muestra que la arquitectura se basa en dos tecnologías importantes, el Internet de las Cosas y sistemas de identificación por radiofrecuencia. Además se identificó que la mayoría de los sistemas inteligentes basados en el Internet de las Cosas, deben considerar 5 capas o fases para administrar y controlar el inventario de forma inteligente.

\section{Palabras Clave:}

Administración de inventarios, Internet de las Cosas, RFID

\footnotetext{
a Universidad Autónoma del Estado de Hidalgo, https://orcid.org/0000-0002-9821-0369, Email: or399260@uaeh.edu.mx,

b Universidad Autónoma del Estado de Hidalgo, https://orcid.org/0000-0002-1519-953X, Email: ve398250@uaeh.edu.mx.

c Universidad Autónoma del Estado de Hidalgo, https://orcid.org/0000-0002-6790-3170 Email: he402426@uaeh.edu.mx

d Autor de Correspondencia, Universidad Autónoma del Estado de Hidalgo, https://orcid.org/0000-0003-2805-6674, Email: igonzalez@uaeh.edu.mx

e Universidad Autónoma del Estado de Hidalgo, https://orcid.org/0000-0002-1997-5399, Email: jorge_zuno_@uaeh.edu.mx
} 


\section{Introducción}

Hoy en día, las empresas se enfrentan a un mercado más competitivo y tecnológico (el comercio a través del ecomercio y la Industria 4.0). Debido a esta competencia, la administración de la cadena de suministro representa uno de los factores más críticos en la rentabilidad de un negocio. En un mercado tan complejo, el diseño, la integración y la operación de la cadena de suministro son elementos estratégicos para producir y distribuir un producto (bienes) o servicio. Esta complejidad ha provocado que las empresas se enfrenten al desafío de identificar, seguir, controlar y mejorar el rendimiento de todos los procesos de la cadena. Por lo tanto, la importancia de la eficiencia de la cadena de suministro como ventaja competitiva ha generado retos significativos para el desarrollo de cadenas más robustas.

Granillo-Macías et al. (2019); Ivanov, Tsipoulanidis y Schönberger (2017) mencionan que la cadena de suministro es una red de organizaciones y procesos en la que varias empresas (proveedores, fabricantes, distribuidores y minoristas) colaboran (cooperan y coordinan) a lo largo de toda la cadena de valor para adquirir materias primas, después convertir estas materias primas en productos o servicios y finalmente entregar estos productos o servicios a los clientes. Por otra parte, el término "Administración de la Cadena de Suministro" actualmente es considerado como un principio de integración y coordinación entre departamentos y empresas para planificar, administrar y controlar todas las actividades de la cadena con el objetivo de maximizar el valor al cliente y lograr una ventaja competitiva sostenible (Ivanov, Tsipoulanidis y Schönberger 2017; Zijm et al. 2019).

Entre las diferentes actividades de la cadena de suministro, la administración de almacenes juega uno de los papeles más importantes, ya que proporcionan la información central para las funciones de entrada (recepción de materia prima o productos, transferencia de los materiales o productos al almacén, identificación de productos o materia prima, clasificación y envío a sus ubicaciones asignadas) y funciones de salida (recolección, empacar, enviar y actualizar el registro). A través de estas instalaciones, los almacenes proporcionan economías de escala ya que las operaciones de almacenamiento permiten actuar como amortiguador, equilibrando la oferta y demanda a corto y largo plazo (González-Hernández et al. 2019; Jabbar et al. 2018).

Debido a la dinámica actual del comercio, las operaciones de alta velocidad en los almacenes se han convirtiendo en las principales actividades a optimizar. Ya que, las dificultades para admin istrar los almacenes y el inventario en entornos operativos complejos se debe a la incertidumbre o aleatoriedad de los diversos procesos comerciales entrelazados, dado que es difícil monitorear, recopilar y procesar la información necesaria para tomar decisiones en tiempo real (Zhou et al. 2017).

Bajo este contexto, Soheilirad etal. (2018) mencionan que desde la década de 1990, la administración de la cadena de suministro ha evolucionado rápidamente mostrando un crecimiento exponencial en trabajos publicados en diferentes revistas (admin istración y tecnología) de interés para académicos y profesionales. Las investigaciones en esta área se han centrado principalmente en mejorar la eficiencia y la ventaja competitiva de los involucrados. Desde la perspectiva de Jabbar et al. (2018), mencionan que para enfrentar los desafíos del mercado globalizado altamente competitivo de hoy, la incorporación de tecnologías emergentes de la Industria 4.0 en los procesos de logística y cadena de suministro pueden contribuir activamente a acelerar las soluciones relacionadas con las operaciones, la productividad y la rentabilidad.

Autores como Jabbar et al. (2018); Ma (2019); Nayyar, Nguyen y Nguyen (2020) mencionan que las tecnologías impulsadas por la Industria 4.0 como el Internet de las Cosas, sistemas Identificación por Radiofrecuencia (RFID, por sus siglas en inglés), sensores de identificación automática, vehículos autónomos, vehículos aéreos no tripulados, realidad aumentada y simulación, han creado un entorno ideal para la implementación e integración de estas tecnologías en la administración de almacenes para transformar las operaciones con el objetivo de maximizar la productividad del almacén y minimizar el costo de las operaciones.

Por otra parte, Elbasani, Siriporn y Choi (2020) mencionan que, en la cuarta revolución industrial, los sistemas RFID y sensores son considerados la principal fuente de generación de datos en entornos inteligentes. Desde el contexto de este trabajo, los entornos inteligentes son: fabricación inteligente y sistemas de administración de almacenamiento inteligentes, los cuales utilizan Internet de las Cosas. Los au tores también describen en su trabajo que, entre las tecnologías del Internet de las Cosas y los sistemas RFID se están u tilizando ampliamente como una inversión de bajo costo económico y con una poderosa capacidad para identificar, trazar y rastrear materiales, productos u objetos físicos, proporcionando información (datos) en tiempo real, disminuyendo los errores humanos, simplificando procesos, aumentando la precisión sobre el almacenamiento y mejorando la eficiencia de los almacenes.

De tal forma que, basado en la necesidad de mejorar la productividad en la cadena de valory motivado porel tema de adopción de tecnologías de la Industria 4.0 en la administración de almacenes. En este trabajo se presenta una investigación cualitativa, de carácter bibliográfico y documental para identificar los principales componentes 
de una arquitectura para la administración y control de inventarios de forma inteligente utilizando las tecnologías impulsadas por la Industria 4.0, como el Internet de las Cosas y Sistemas de Identificación por Radiofrecuencia.

\section{Trabajos relacionados}

El objetivo principal de cualquier solución tecnológica en la administración de los almacenes debe proporcionar transparencia y control de la información, la trazabilidad y localización de los materiales y/o productos en tiempo real para garantizar la entrega de los materiales a las líneas de producción o la entrega de productos en el momento, lugar, cantidad, condición y costos correctos. Por lo tanto, se requiere de un seguimiento inteligente de los materiales o productos en toda la cadena de suministro para facilitar a los administradores la toma de decisiones y maximizarel nivel de servicio al cliente (Anandhi, Anitha y Sureshkumar 2019).

Por ejemplo, H. Liu et al. (2019) realizaron un análisis de las características y ventajas de la aplicación de tecnología RFID y de sensores para el diseño de un layout de un nuevo almacén con el objetivo de resolver efectivamente los problemas existentes en la administración de un almacén. Alyahya, Wang y Bennett (2016) presentaron una investigación sobre una metodología en la que un sistema de administración del inventario basado en RFID tiene la capacidad de ani (2015) propusieron una infraestructura del Internet de las Cosas basada en RFID, inteligencia ambiental y un sistema de agentes múltiples para el cumplimiento de pedidos en un sistema de almacenamiento colaborativo. Esta infraestructura consistió en una capa de dispositivos físicos, una plataforma ambiental de middleware, un sistema de múltiples agentes y un sistema de planeación de recursos empresariales.

J. Liu et al. (2018) propusieron un sistema de administración de almacenes basado en sistemas RFID, servicio ONS, servicio PML, tecnología GIS, tecnología GPS e Internet de las Cosas. También Trab et al. (2018) proponen un sistema de administración de almacenes habilitado con RFID e Internetde las Cosas con el objetivo de determinar las ubicaciones de almacenamiento adecuadas mediante un método de almacenamiento basado en la clase de producto.

Pang et al. (2019) proponen un diseño de para una arquitectu ra o esquema de un sistema de gestión logística basado en RFID. Los autores toman RFID como núcleo, combinando con NB-loT y tecnologías de aprendizaje profundo, de tal forma que, proponen una solución logística inteligente, que incluye seguimiento logístico, clasificación auxiliar, carga y descarga rápida, posicionamiento de carga e inventarioy recolección para un sistema de inventarios. interactuar con un mecanismo de recuperación y almacenamiento automático habilitado con RFID sin ninguna intervención humana.

Wei y Leung (2011) abordaron un problema de la administración de almacenes con el objetivo de diseñar estrategias de carga utilizando información proporcionada por la tecnología RFID. Para elegirlas mejores estrategias de carga realizaron modelos de simulación para comparar el proceso de carga actual con los modelos propuestos con RFID para probar que modelos funcionan mejor en términos de tiempos de carga y el porcentaje de camiones cargados en tiempo y forma. También Giubilato, Zhang y Alfieri (2019) utilizaron un modelo de simulación de eventos discretos para investigar cómo el RFID puede reducir el impacto de la inexactitud de inventario en la cadena de suministro de contenedores retornables de la industria au tomotriz.

Han, Bae, and Lee (2018) propusieron una arquitectura para la auditoría y el seguimiento continuo del inventario en un entorno al aire libre utilizando tecnologías como: vehículos aéreos no tripulados (conocido comúnmente como "dron"), un transmisor de señal basado en iBeacon e Internet de las Cosas, con el objetivo de que la información sobre el inventario se actualice en tiempo real, de tal forma que, el sistema pueda detectar automáticamente la ubicación de los artículos del inventario y avisar con anticipación de cualquier cuello de botella en el suministro. Reaidy, Gunasekaran y Spalanz

\section{Arquitectura para la administración de almacenes basado en Internet de la Cosas y RFID}

Desde el contexto de la administración de la cadena de suministro el Internet de las Cosas se puede definir como el conjunto de objetos físicos que están conectados digitalmente para detectar, monitorear e interactuar entre los departamentos de una empresa, y entre la empresa y su cadena de suministro (proveedores y clientes) consolidando la agilidad, la visibilidad y el intercambio y seguimiento de la información para facilitar el plan, el control y la coordinación de los procesos de la cadena de suministro (Abdel-Basset, Manogaran y Mohamed 2018). La RFID es una tecnología clave ampliamente utilizada que se considera un requisito previo o un elemento esencial en el Internet de las Cosas. Se compone principalmente de tres elementos: una etiqueta formada por un chip conectado con una antena, un lector que emite señales de radio y recibe a cambio respuestas y un middleware que une el hardware RFID y las aplicaciones empresariales (Fan et al. 2015), como se muestra en la Figura 1. 

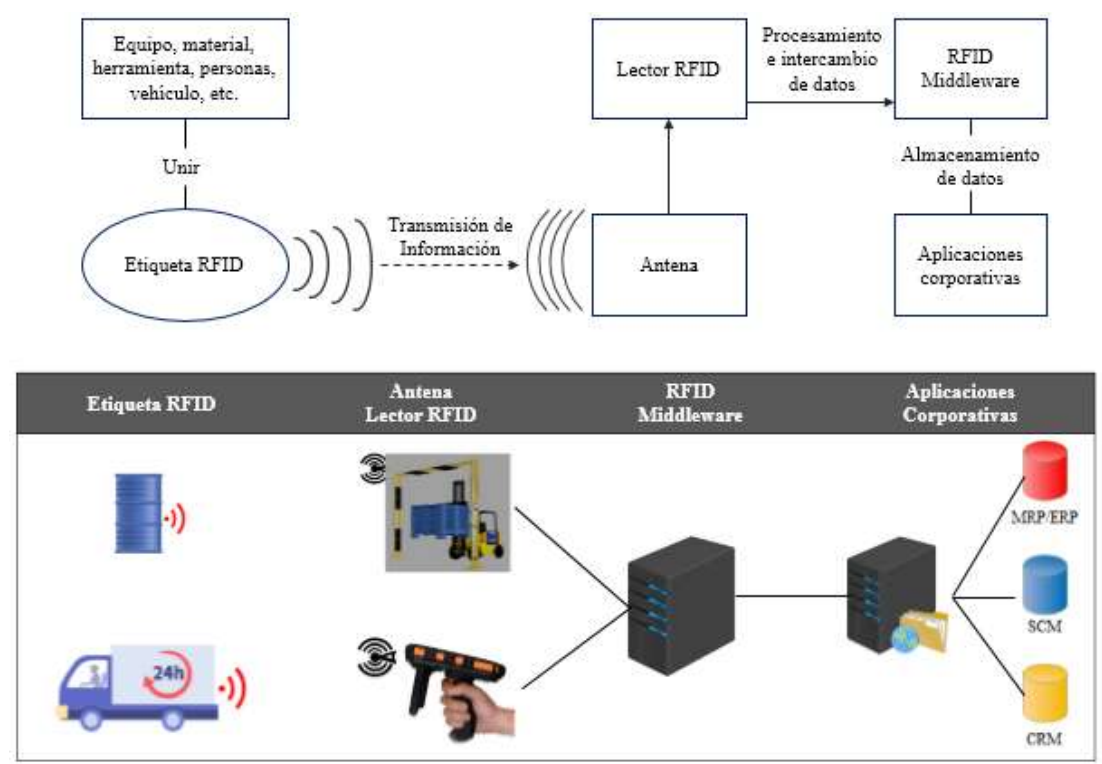

Figura 1. Componentes RFID

La tecnología RFID en sí misma es simple, se puede encontrar en formato de largo y bajo alcance. Un RFID de largo alcance utiliza una banda de "frecuencia ultra alta" (UHF, por sus siglas en inglés), la cual tiene la velocidad de reconocimiento más rápida y puede leer etiquetas de hasta 15 metros de distancia, además es adecuada para su uso en industrias y almacenes. EI RFID de bajo alcance utiliza una banda de "alta frecuencia" (HF, por sus siglas en inglés) que puede proporcionar una de lectura de etiquetas de hasta 1.2 metros de distancia (Elbasani, Siriporn y Choi 2020).

Esta tecnología de identificación automática desempeña un papel estratégico en la administración de almacenes en el enlace del flujo de información (datos) de los procesos y la información (datos) de los activos físicos. Los beneficios clave del despliegue de sistemas RFID en la cadena de suministro incluyen el seguimiento del producto, la transparencia de la información, la reducción del efecto látigo, la reducción de la inexactitud del inventario causada por la contracción y los errores de ubicación incorrecta, mejora de la gestión del ciclo de vida del producto, reduce de los costos laborales y aumenta la flexibilidad (Drakaki y Tzionas 2019; Elbasani, Siripom y Choi 2020).

Por lo tanto, el Internet de las Cosas es una interconexión de dispositivos masivos en el ciberespacio mediante la recopilación, el intercambio y el análisis de datos en una red heterogénea. El Internet de las Cosas proporciona servicios a través del Internet tradicional al permitir comunicaciones de persona a cosa, de cosa a cosa o de cosa a cosas. De tal forma que, el núcleo de esta tecnología se encuentra en los sistemas integrados con etiquetas RFID, sensores y actuadores que pueden interconectarse entre sí con objetivos comunes.

Considerando lo anterior, la arquitectura del Internet de las Cosas se define como un marco para especificar los componentes físicos de una red, su organización y configuración funcional, sus principios y procedimientos operativos, y los formatos de datos utilizados en su funcionamiento. Con base en los autores Hemalatha y Afreeen (2015); dos Santos y Canedo (2019); Wang et al. (2015), la Figura 2 muestra en forma general la arquitectura de un sistema de administración de almacenes basado en Internet de las Cosas y RFID, en donde se consideran 5 capas en la arquitectura: capa física, capa de red, capa de middleware, capa de aplicación y capa empresarial.

- Capa Física: la capa física también se conoce como capa de dispositivo. Se compone de objetos físicos y dispositivos que tienen como objetivo recopilar y procesar información. Los dispositivos pueden ser identificación por radiofrecuencia (RFID), código de barras, sensor de infrarrojos, según sea el método de identificación de los objetos. Básicamente, esta capa se ocupa de la identificación y recopilación de información específica del objeto por parte de los dispositivos. La información recopilada se envía a la capa de red.

- Capa Red: la capa de red también se conoce como la capa de transmisión, la cual transfierelos datos producidos por la capa física a la capa de middleware a través de canales seguros. El medio de transmisión puede ser cableado o 
inalámbrico y la tecnología puede ser $3 \mathrm{G} / 4 \mathrm{G}$, GSM, UMTS, WiFi, Bluetooth de baja energía, infrarrojos, ZigBee, etc. Además, otras funciones como la computación en la nube y los procesos de gestión de datos se manejan en esta capa.

- Capa de middleware: los dispositivos del Intemet de las Cosas implementan diferentes tipos de servicios. Cada dispositivo se conecta y se comunica sólo con otros dispositivos que implementan el mismo tipo de servicio. Esta capa permite a los programadores de aplicaciones del Internet de la Cosas trabajar con objetos heterogéneos sin tener en cuenta una plataforma de hardware específica. Además, esta capa procesa los datos recibidos, toma decisiones y brinda los servicios requeridos a través de los protocolos de conexión de red. Es decir, esta capa es responsable de administrar los servicios $y$ tiene un enlace a la base de datos, de tal forma que, realiza el procesamiento de información, computación ubicua y toma decisiones automáticas basadas en los resultados.

- Capa de aplicación: esta capa proporciona los servicios solicitados por los clientes y una administración global de dispositivos basada en la información de los objetos procesados en la capa de middleware. La importancia de esta capa para el Internet de las Cosas es que tiene la capacidad de proporcionar servicios inteligentes de alta calidad para satisfacer las necesidades de los clientes. La capa de aplicación cubre numerosos mercados como el hogar inteligente, el edificio inteligente, transporte inteligente, automatización y producción industrial inteligente, atención médica inteligente, agricultura inteligente, ciudad inteligente, etc.

- Capa empresarial: esta capa es responsable de administrar el sistema del Internet de las Cosas de forma general, incluidas las aplicaciones, actividades y los servicios. Las responsabilidades de esta capa son construir un modelo de negocios, gráficos, diagramas de flujo, etc., basados en los datos recibidos de la capa de aplicación. También se supone que diseña, analiza, implementa, evalúa, supervisa y desarrolla elementos relacionados con el sistema del Internet de las Cosas. La capa empresarial permite apoyar los procesos de toma de decisiones basados en el análisis de Big Data. Además, el monitoreo y la gestión de las cuatro capas subyacentes se logran en esta capa. Además, esta capa compara la salida de cada capa con la salida esperada para mejorar los servicios y mantener la privacidad de los usuarios.

El Internet de las Cosas permite crear muchas aplicaciones en diferentes dominios de aplicación. dos Santos y Canedo (2019) mencionan que el dominio de la aplicación se puede dividir principalmente en tres categorías según su enfoque: industria, medio ambiente y sociedad. Por lo tanto, las aplicaciones de administración y control de inventarios, transporte y logística, aeroespacial, aviación y automotriz son algunas de las aplicaciones enfocadas en la industria. Telecomunicaciones, tecnología médica, atención médica, construcción inteligente, hogar y oficina, medios, entretenimiento y venta de entradas son algunas de las aplicaciones enfocadas en la sociedad. Agricultura, reciclaje, advertencia de desastres, monitoreo ambiental son algunas de las aplicaciones enfocadas en el medio ambiente.

Desde el contexto de la cadena de suministro y la logística, la precisión de la información (datos) del inventario puede ser crucial para las operaciones de almacén, para la planeación de la producción y para la administración y control de inventario (materia prima o productos) debido a las entradas y salidas constantemente de la materia prima o producto terminado. Durante la última década, se ha visto un fuerte aumento de las actividades del e-comercio, ahora, muchos clientes prefieren comprarproductos en lín ea y exigen una entrega rápida de los productos solicitados que se despacharán directamente en la puerta de su casa. Por lo tanto, existe la necesidad de que estos sectores busquen métodos aún más eficientes y efectivos para almacenar, recoger y enviar mercancías en centros de distribución cada vez más centralizados en los que la automatización e integración de los sistemas de almacenamiento es inevitable. Por lo tanto, la administración de almacenes y el control de inventarios se está desarrollando hacia la dirección de sermás inteligente para lograrel propósito de la integración y distribución bajo demanda, manejar el procesamiento y la gestión de la información de decenas de miles de pedidos todos los días, para completar el acoplamiento del flujo de negocios y la logística.

Bajo este panorama, la infraestructura del Internet de las Cosas asociada con la tecnología RFID en la administración y control de inventarios está desempeñando un papel importante como un medio de comunicación y un proveedor de datos para hacer más eficiente los procesos y minimizar los costos de la cadena de suministro. 


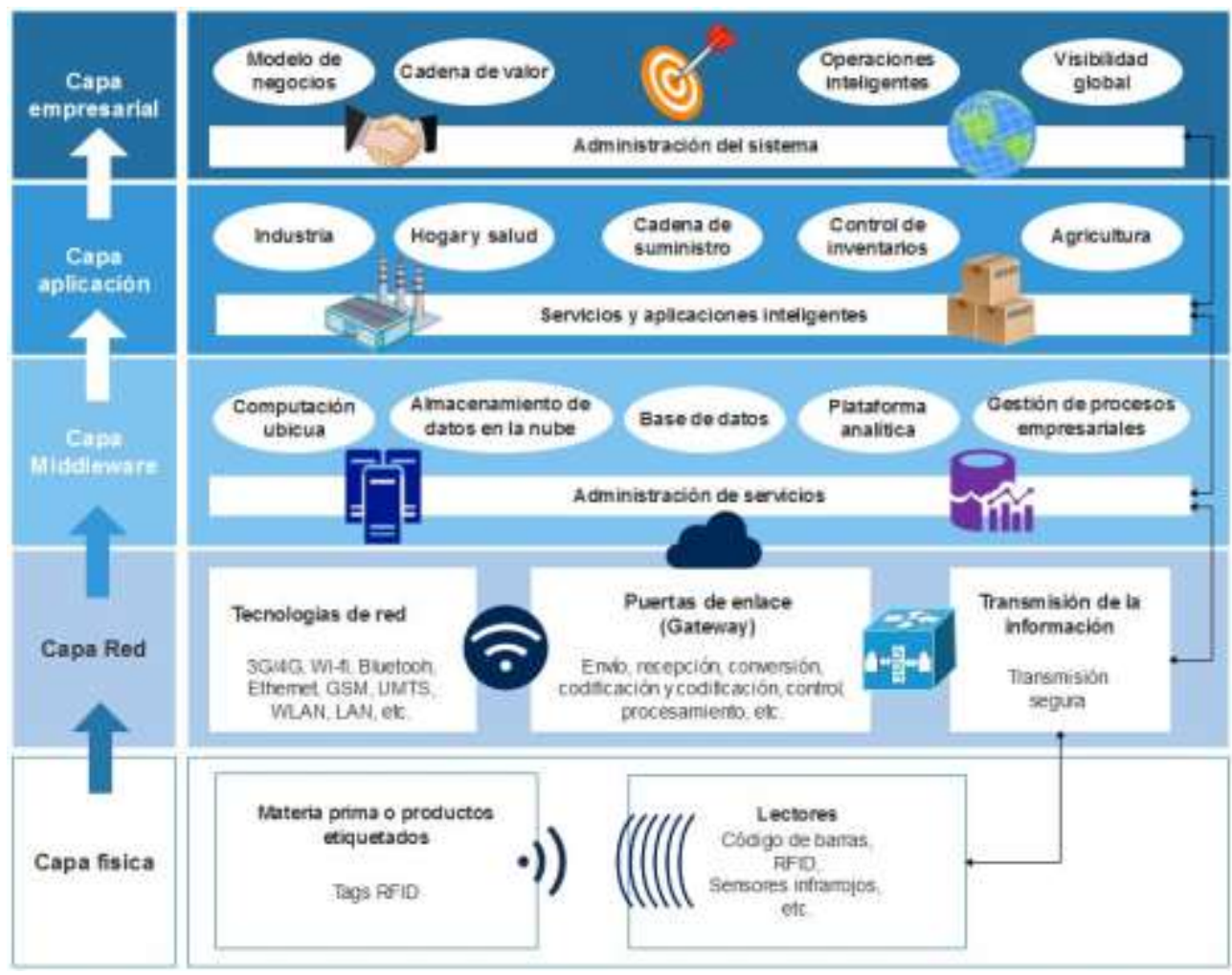

Figura 2. Arquitectura para el Internet de las Cosas

En este sentido la aplicación de esta tecnología en un almacén se centra básicamente en colocar un lector RFID en las puertas de entrada y salida de un almacén que recopila información de la materia prima o producto terminado, los cuales están etiquetados con Tags RFID (etiquetas RFID). Para el proceso de entrada, una vez que el sistema identificó la materia prima o producto que entró al almacén, indica cual debe ser la ubicación de almacenamiento. La información recopilada por el lector sobre el inventario, se actualiza instantáneamente en un sistema de administración del almacén a través de un controlador que transmite los datos recopilados a través de un middleware. El middleware es la capa de traducción de software entre un lector RFID y el sistema de administración de almacenes. La base de datos del sistema de administración del almacén contiene registros, que incluyen datos de identificación, disponibilidad y otra información definida por el uso de cada artículo almacenado en el almacén. Una vez que se ordena una materia prima o un artículo, el sistema de administración del inventario basado en RFID tiene la capacidad de llevar a cabo una verificación automática de los datos de información de la materia prima o producto en la base de datos. Una vez que el artículo ordenado es identificado por el sistema de administración del inventario basado en RFID, se recopila o recoge en su posición de almacenamiento, ya sea de forma manual o automatizada. Finalmente la base de datos del inventario del almacén basada en RFID se actualizará tan pronto como la materia prima o el producto se retire del almacén en un transporte que pase por la puerta de entrada/salida del almacén.

Finalmente, después de realizar una investigación cualitativa, de carácter bibliográfico y documental se puede inferir que la administración y control de inventanios basada en el Internet de las Cosas y RFID da como resultado:

- La optimización de procesos logísticos.

- Optimiza los costos operativos.

- Minimiza trabajos manuales.

- Reduce tiempos de operación en los almacenes.

- Genera un mayor control del inventario (aumente la visibilidad en los niveles de existencias).

- Recopila y procesa datos (información) en tiempo real.

- Genera mayor presión de la información sobre la trazabilidad y rastreo de la materia prima o productos.

- Aumenta la seguridad de los trabajadores.

- Aumenta la productividad. 


\section{Conclusión}

En la cadena de suministro y los sectores logísticos, la precisión de los datos del inventario es esencial, ya que esta información o datos puede ser cruciales para las operaciones de almacén, la planeación de la producción, la administración y el control del inventario entrante y saliente, así como todos los procesos que se desarrollan en un almacén. Este documento se centró una investigación cualitativa, de carácter bibliográfico y documental para identificar los componentes básicos de una arquitectura para integrar el Internet de las Cosas y RFID en la administración y control de inventarios. De acuerdo con los autores citados las principales capas de una arquitectura para la administración del inventario deben considerar las siguientes capas: capa física, capa de red, capa de middleware, capa de aplicación y capa empresarial. En donde la tecnología RFID es considerada la principal fuente de generación de datos en entomos inteligentes.

\section{Referencias}

Abdel-Basset, M., Manogaran, G. y Mohamed, M. (2018). Internet of Things (IoT) and its impact on supply chain: A framework for building smart, secure and efficient systems. Future Generation Computer Systems, 86, 614-628.

Alyahya, S., Wang, Q. y Bennett, N. (2016). Application and integration of an RFID-enabled wa rehousing management system - a feasibility study. Journal of Industrial Information Integration, 4, 15-25.

Anandhi, S., Anitha, R. y Sureshkumar, V. (2019). IoT Enabled RFID Authentication and Secure Object Tracking System for Smart Logistics. Wireless Personal Communications, 104,543-560.

dos Santos, Y. L. y Canedo, E. D. (2019). On the design and implementation of an IoT based architecture for reading ultra high frequency tags. Information, 10(2), 1-17.

Drakaki, M. y Tzionas, P. (2019). Investigating the impact of inventory inaccuracy on the bullwhip effect in RFID-enabled supply chains using colored petri nets. Journal of Modelling in Management, 14(2), 360384 .

Elbasani, E., Siriporn, P. y Choi, J. S. (2020). A Survey on RFID in Industry 4.0. In G. R. Kanagachidambaresan, R. Anand, E. Balasubramanian y V. Mahima (Eds.), Internet of Things for Industry 4.0: Design, Challenges and Solutions (pp. 1-16). Springer.

Fan, T., Tao, F., Deng, S. y Li, S. (2015). Impact of RFID technology on supply chain decisions with inventory inaccuracies. International Journal of Production Economics, 159, 117-125.

Giubilato, B., Zhang, G. y Alfieri, A. (2019). Automotive Returnable Container Management with RFID: A Simulation Approach. IFAC PapersOnLine, 52(13), 355-360. Elsevier Ltd.

González-Hernández, I. J., Martínez-Flores, J. L., Sánchez-Partida, D. y Gibaja-Romero, D. E. (2019). Relocation of the distribution center of a motor oil producer reducing its storage capacity: A case study. Simulation, 95(11), 1097-1112.

Granillo-Macías, R., González-Hernández, I. J., Martínez-Flores, J. L., Caballero-Morales, S. O. y Olivares-Benitez, E. (2019). Hybrid model to design a distribution network in contract farming. DYNA, 86(208), 108-114.
Han, K. H., Bae, S. M. y Lee, W. (2018). Integrated Inventory Management System for Outdoors Stocks Based on Small UAV and Bea con. In Á. Rocha, H. Adeli, L. P. Reis y S. Costanzo (Eds.), Trends and Advances in Information Systems and Technologies. WorldCIST'18 2018. Advances in Intelligent Systems and Computing(vol746, pp. 533-541). Springer.

Hemalatha, D. y Afreeen, B. (2015). Development in RFID (Radio Frequency Identification) Technology in Internet of Things (IOT). International Journal of Advanced Research in Computer Engineering \& Technology (IJARCET), 4(11), 4030-4038.

Ivanov, D., Tsipoulanidis, A. y Schönberger, J. (2017). Global Supply Chain and Operations Management: A Decision-Oriented Introduction to the Creation of Value. Springer.

Jabbar, S., Khan, M., Silva, B. N. y Han, K. (2018). A REST-based industrial web of things' framework for smart wa rehousing. The Journal of Supercomputing, 74(9), 4419-4433.

Liu, H., Yao, Z., Zeng, L. y Luan, J. (2019). An RFID and sensor technology-based warehouse center: assessment of new model on a superstore in China. Assembly Automation, 39(1), 86-100.

Liu, J., Li, Z., Sun, L., Wang, J. y Cao, N. (2018). Research on the Application of RFID Technology in Logistics Warehousing Management System. In T. Hu, F. Wang, H. Li, \& Q. Wang (Eds.), Algorithms and Architectures for Parallel Processing. ICA3PP 2018. Lecture Notes in Computer Science (vol 11338, pp. 158-164). Springer.

Ma, H. (2019). An Industry 4.0 Technologies-Driven Warehouse Resource Management System. In K. Wang, Y. Wang, J. O. Strandhagen y T. Yu (Eds.), Advanced Manufacturing and Automation VIII. IWAMA 2018. Lecture Notes in Electrical Engineering (Vol. 484, pp. 27-33). Springer, Singapore.

Nayyar, A., Nguyen, B.-L. y Nguyen, N. G. (2020). The Internet of Drone Things (IoDT): Future Envision of Smart Drones. In A. K. Luhach, J. A. Kosa, R. C. Poonia, X.-Z. Gao y D. Singh (Eds.), First International Conference on Sustainable Technologies for Computational Intelligence. Advances in Intelligent Systems and Computing (vol 1045, pp. 563-580). Singapore: Springer.

Pang, J., Shen, L., Zhang, Q., Xu, H. y Li, P. (2019). Design of Modem Logistics Management System Based on RFID and NB-IoT. In L. Barolli, M. Takizawa, F. Xhafa y T. Enokido (Eds.), Web, Artificial Intelligence and Network Applications. WAINA 2019. Advances in Intelligent Systems and Computing (Vol. 927, pp. 561-569).

Reaidy, P. J., Gunasekaran, A. y Spalanzani, A. (2015). Bottom-up approach based on Internet of Things for order fulfillment in a collaborative warehousing environment. International Journal of Production Economics, 159, 29-40.

Soheilirad, S., Govindan, K., Mardani, A., Zavadskas, E. K., Nilashi, M. y Zakuan, N. (2018). Application of data envelopment analysis models in supply chain management: a systematic review and meta-analysis. Annals of Operations Research, 271(2), 915-969.

Trab, S., Bajic, E., Zouinkhi, A., Naceur Abdelkrim, M. y Chekir, H. (2018). RFID IoT-enabled warehouse for safety management using product class-based storage and potential fields methods. International Journal of Embedded Systems, 10(1), 71-88.

Wang, M., Tan, J., Wang, M. y Li, Y. (2015). Design and implementation of enterprise a sset management system based on IOT technology. 2015 IEEE International Conference on Communication Software and Networks (ICCSN), 384-388.

Wei, J. y Leung, S. C. H. (2011). A simulation modeling and analy sis for RFID-enabled mixed-product loading strategy for outbound logistics: A case study. Computers and IndustrialEngineering, 61(1), 209-215.

Zhou, W., Piramuthu, S., Chu, F. y Chu, C. (2017). RFID-enabled flexible warehousing. Decision Support Systems, 98, 99-112.

Zijm, H., Klumpp, M., Heragu, S. y Regattieri, A. (2019). Operations, Logistics and Supply Chain Management: Definitions and Objectives. In H. Zijm, M. Klumpp, A. Regattieri y S. Heragu (Eds.), Operations, Logistics and Supply Chain Management (pp. 27-42). Springer. 